\title{
Literatur
}

Csáky, Moritz (2010): Das Gedächtnis des Städte. Kulturelle Verflechtungen - Wien und die urbanen Milieus in Zentraleuropa. Wien.

Hlobil, Tomáš (2012): Geschmacksbildung im Nationalinteresse. Die Anfänge der Prager Universitätsästhetik im mitteleuropäischen Kulturraum 1763-1805, aus dem Tschechischen übersetzt von Jürgen Ostmeyer. Hannover.

Hlobil, Tomáš (2018): Geschmacksbildung im Nationalinteresse II. Der Abschluss der frühen Prager Universitätsästhetik im mitteleuropäischen Kulturraum 1805-1848, aus dem Tschechischen übersetzt von Jürgen Ostmeyer. Hannover.

Kontantinović, Zoran / Rinner, Fridrun (2003): Eine Literaturgeschichte Mitteleuropas. Innsbruck.

Kriegleder, Wynfrid / Seidler, Andrea / Tancer, Jozef (Hrsg.) (2019): Kulturelle Zirkulation im Habsburgerreich. Der Kommunikationsraum Wien. Wien.

Naumann, Friedrich (1915): Mitteleuropa. Berlin.

Perloff, Marjorie (2016): Edge of Irony. Modernism in the Shadow of the Habsburg Empire. Chicago. Puchalski, Lucjan (2011): Edward Lubomirskis Wien-Erfahrung zwischen eigenem und fremdem Kulturgedächtnis. In: Golec, Janusz / Lühe, Irmela von der (Hrsg.): Geschichte und Gedächtnis in der Literatur vom 18. bis 21. Jahrhundert. Frankfurt am Main. S. 83-94.

Lucjan Puchalski

(Universität Wrocław, Wrocław) ORCID: 0000-0002-3139-4925

Lucjan Puchalski, Uniwersytet Wrocławski, Instytut Filologii Germańskiej, pl. biskupa Nankiera 15b, 50-140 Wrocław, Polen, E-Mail: lucjan.puchalski@uwr.edu.pl.

Received: 30.09.2020, accepted: 1.03.2021

\section{Reise- und Medienkultur im deutsch-polnischen Kontext in der Frühen Neuzeit}

Joanna Kodzik, Anna Mikołajewska (Hrsg.): Reisen, wahrnehmen, kommunizieren im deutsch-polnischen Kontext in der Frühen Neuzeit. Festschrift zum 65. Geburtstag von Prof. Dr. Włodzimierz Zientara, mit einem Vorwort von Holger Böning, Edition Lumiére, Bremen 2019, 317 S.

https://doi.org/10.19195/0435-5865.146.14

Schreiben bei Gelegenheit ist als eine Kulturpraktik in der Frühen Neuzeit, die genuine sowohl zum Zeremonialwesen als auch zu den Ritualen der Gelehrtenwelt gehörte, den Barockforschern gut bekannt. ${ }^{1}$ Der vorliegende Band wurde zu einer festlichen occasio, nämlich zum 65. Jubiläum des Thorner Germanisten und Frühneuzeitforschers, Prof. Dr. hab. Włodzimerz Zientara, verfasst und reiht sich sehr wohl in diese Tradition hinein. Seine Struktur entspricht den Hauptbereichen der wissenschaftlichen Interessen und For-

1 Segebrecht 1977. 
schungen des Jubilaren und zeigt ein gut durchdachtes Konzept der kultur- und mediengeschichtlich, sowie mikrohistorisch perspektivierten Beiträge dar, in denen Germanisten und Historiker folgende Forschungsfelder kontextualisieren: 1. Deutsch-polnische Kulturbeziehungen in der Frühen Neuzeit, 2. Die barocke Reisekultur und das Phänomen des Fremden, 3. Die Medienkultur der Epoche - vor allem Pressegeschichte. Ich werde nicht das ganze thematische Spektrum des Bandes berücksichtigen, sondern mich auf ausgewählte Aspekte konzentrieren, die meinem eigenen Forschungsinteresse entsprechen. Einerseits gilt meine Aufmerksamkeit jenen Texten, die es erlauben, in einer komparatistischen Sicht die literarischen und kulturgeschichtlichen Phänomene der Epoche im Königlichen Preußen und in Schlesien zu reflektieren. Dabei können nicht nur Schnittpunkte der mikrohistorischen Entwicklungen dieser beiden Regionen, sondern auch Forschungslücken sichtbar gemacht werden, die schnellstens zu schließen wären. Andererseits werde ich auf Beiträge eingehen, die alltagsgeschichtliche Aspekte des Lebens und Reisens im 17. Jahrhundert thematisieren und somit für die anthropologisch ausgerichtete Barockforschung interessant erscheinen. Schlesien war, wie es sich gleich im ersten Beitrag des Bandes herausstellt, das Territorium im Reich, aus dem die Migration nach Königlich Preußen und d.h. nach Thorn und die Umgebung bereits im 13. Jahrhundert erfolgte: ,,[In] der ersten Hälfte des 13. Jahrhunderts“ - schreibt Krzysztof Kopiński - ,stammten die meisten Thorner Stadtbürger aus Schlesien". ${ }^{2}$ Im 15. Jahrhundert, als Thorn an seiner Bedeutung zusehends verlor, begann die Auswanderung der Patrizier sowohl nach Danzig als auch nach Breslau, der Odermetropole, die damals als Zentrum europäischen Handels und Gewerbes gerade aufblühte. ${ }^{3}$ Eine Studie über die Rolle Schlesiens in diesem komplizierten Migrationsprozess steht noch aus. Das gleiche gilt allerdings auch für eine eingehende Untersuchung der gegenseitigen Beziehungen auf dem Gebiet der institutionellen Bildung. Schlesien war durch das Fehlen einer Universität bis 1702 eine europäische Metropole zweiten Ranges, ${ }^{4}$ aber dieses Defizit wurde sehr bald durch hervorragende Gymnasien in Goldberg, Brieg und vor allem durch das Elisabethanum und das Magdaleneum in Breslau nachgeholt. Nichtdestotrotz zogen im Laufe des 17. Jahrhunderts auch die akademischen Gymnasien in Thorn und Danzig, die sich in ganz Europa eines Rufes illustrer Bildungsinstitutionen erfreuten, schlesische Schüler an, mit Andreas Gryphius und Christian Hoffmann von Hoffmannswaldau an der Spitze. ${ }^{5} \mathrm{Zu}$ meinem Bedauern fand sich in dem Jubiläumsband kein Beitrag zur Geschichte und kulturellen Rolle des Thorner Gymnasiums im 17. Jahrhundert.

Seit der profunden Studie von Włodzimierz Zientara: „Sarmatia Europiana oder Sarmatia Asiana?"6 wird der Reisediskurs der Frühen Neuzeit um die osteuropäische Perspektive signifikant bereichert. Es geht dabei nicht nur um Reisekultur der Europäer, die durch stereotype Vorstellungen über das rückständige, durch Kälte, Schmutz, mangelnde Hygiene, geringe Anzahl von akzeptablen Gasthäusern unangenehm/unatraktiv erscheinende Polen bestimmt war, sondern auch um alltagsgeschichtliche Reisebedingungen nach und in Polen-Litauen, denen im besprochenen Band mehrere Beiträge gewidmet sind. Eben-

${ }^{2}$ Kopiński 2019: 19-28, hier S. 19.

3 Czarnecka 2012: 197-238.

${ }^{4}$ Lambrecht 2000: 117-141, hier. S. 117.

5 Szyrocki 1964: 20-24; Wotschke 1939: 196-216; Zientara 1996: 215-222. Vgl. auch: Czubański / Kulak 1987.

6 Zientara 2003. 
falls interessant zu lesen sind die Beschreibungen Jarosław Dumanowskis über altpolnische Kochrezepte aus dem 16.-18. Jahrhundert, die vor allem praktische Hinweise für Bearbeitung und Konservierung von Lebensmitteln für die Reise enthalten. ${ }^{7}$ Dieser Beitrag ist eine kleine Kulturgeschichte des Bouillontäfelchen, das in den Kochrezepten aus dem 17. Jahrhundert als unentbehrliches Nahrungsmittel auf der Reise einen zentralen Platz einnimmt. Axel Walter analysiert gerade die seit dem ausgehenden 16. Jahrhundert erscheinenden deutschen Reiseführer unter dem besonderen Augenmerk auf „Herbergen und Bewirtung ", ${ }^{8}$ was als zentrales Aspekt der Reisekultur in der Frühen Neuzeit schlechthin erscheint. Seine Ausführungen erhalten einen besonderen Wert im Kontext der problematischen Übernachtungsmöglichkeiten auf Reisen durch Osteuropa. Leider hat Walter in seinen Überlegungen Frauen als Mit-Reisende völlig aus dem Blickwinkel verloren. Um eine „angemessene Bewirtung der Gäste“ geht es auch im Beitrag von Edmund Kodzik, der die Besuche auf der Festung Weichselmünde bei Danzig als festes Programm für prominente und hohe Gäste, wie etwa polnische Könige, die königliche Familie, ausländische Diplomaten, kirchliche und staatliche Würdeträger, thematisiert. ${ }^{9}$ Anhand der zitierten Ego-Dokumente wird dieses Programm rekonstruiert: „nach der Vorstellung der Besatzung, einem Spaziergang auf der Mauer und dem Besteigen des Turms wurden die Gäste mit einem Festmahl bewirtet“ (S. 76). Das erwähnte Festmahl kostete die Danziger Kämmerei gewaltige Summen, was Kodzik besonders hervorhebt und damit auf die in der Forschung zu frühneuzeitlichen Reisekultur weniger beachteten, gesellschaftlichen Nebenkosten der ritualisierten Gastfreundschaft rekurriert.

$\mathrm{Zu}$ alltagsgeschichtlichen Aspekten der Reise gehörte genuine die Körperhygiene, die im 17. Jahrhundert - das allgemein als Epoche des Schmutzes und der mangelnden Körperpflege gilt ${ }^{10}$ - in den europäischen Apodemiken generell bemängelt wurde. Im Kontext dieser kritischen Darstellungen pluralisiert Aleksandra Bovt den Körperpflegediskurs der Epoche, indem sie auf positive ggf. begeisterte Berichte der Reisenden in Polen-Litauen verweist, die sogar eine ,intensive Sorge um die Körperpflege, die sich im häufiger als im Westeuropa durchgeführten Baden niederschlug" ${ }^{11}$ registrieren. Zwei Beiträge sind religiös politisch motivierten Reisen im Kontext des Pietismus gewidmet: Joanna Kodzik nennt und analysiert Kommunikationsstrategien auf Missionsreisen der Pietisten aus der Herrnhuter Brüdergemeinde in Polen-Litauen im 18. Jahrhundert, wie etwa unaufdringliche Kontaktaufnahme, Beherrschung der Sprache des Gastlandes, Identitätswechsel durch Annahme einer anderen gesellschaftlichen Rolle oder Rhetorik des Extremen. ${ }^{12}$ Liliana Lewandowska dagegen stellt die Deutschland-Reise des lutherischen Predigers Samuel Schelwig von 1694 (von Danzig über Berlin, Wittenberg, Leipzig, Jena, Helmstedt, Hamburg, Bremen zurück nach Danzig) anhand seines Reisetagebuchs „Itinerarium Antpietisticum" (1695) dar und weist dabei dieses Ego-Dokument, neben seinen wichtigen Aspekten als Reiseführer, primär als einen polemischen Text aus, der die konfessionelle Identität des streng lutherischen Autors mitbestimmt.

\footnotetext{
7 Dumowski 2019: 39-48.

8 Walter 2019: 51-62, hier S. 52.

9 Kizik 2019: 71-78.

10 Viragello 1988.

11 Bovt 2019: 63-70, hier S. 65.

12 Kodzik 2019: 79.
} 
Sechs weitere Beiträge schreiben sich in den Forschungsbereich der Mediengeschichte in der Frühen Neuzeit ein, wobei Olga Fejtovó Prager historische Schreibkalender aus dem 16. Jahrhundert als Medium des tschechisch-polnischen Kultur- und Wissenstransfers, die bis ins 18. Jahrhundert hinein rezipiert wurden, ${ }^{13}$ analysiert. Die anderen Autoren konzentrieren sich dagegen auf die Entwicklung des Pressewesens in der Frühen Neuzeit. Angefangen von den handgeschriebenen Zeitungen in Polen-Litauen, die sich hier - laut Kazimierz Maliszewski ${ }^{14}$ - besonders intensiv im ausgehenden 17. und im 18. Jahrhundert entwickelten und diverses Wissen über Welt, Leute, Religion, Naturerscheinungen und auch Klatschgeschichten vermittelten und popularisierten, werden nachfolgend Zeitungsnachrichten über Konfliktsituationen in Europa und erste Meldungen aus dem Dreißigjährigen Krieg thematisiert. ${ }^{15}$ Holger Böning rekapituliert hier die Entstehungsgeschichte der Presse im Kontext des Kriegsberichtserstattung, wobei er auf einzelne Zeitungen in Leipzig, Straßburg und Wolfenbüttel eingeht. Es wäre interessant diese Zeitungsberichte auch mit den im vergleichbaren Zeitraum in Breslau erschienenen Zeitungen, wie etwa „Wöchentliche Zeitung aus unterschiedlichen Orthen“ oder „Der Breslauer Mercurius“ zu vergleichen. Für Schlesien-Forschung ist der Beitrag von Michael Nagel über die erste deutsch-jüdische Zeitung, die 1771 in Dyhernfurth (Brzeg Dolny), unweit von Breslau erschien, von Interesse. Die jüdische Diaspora war dort so stark, dass sie sich eine eigene Druckerei finanzieren konnte. Diese Zeitung war eine Kompilation aus den - vor allem politischen - Nachrichten, die in anderen deutschen Zeitungen abgedruckt wurden und sie erschien sowohl in deutscher als auch hebräischer Sprache. Da es bis heute lediglich zwei Exemplare dieser Zeitung (Nr. 2 vom 9. Dezember 1771 und Nr. 9 vom 10. Januar 1772) überliefert sind, ist die Erforschung dieser Raritäten aus Perspektive der deutsch-jüdischen Pressegeschichte besonders wichtig. Die zwei letzten Beiträge betreffen Informationsblätter und Fachzeitschriften der Aufklärungszeit, die wie „Thornische Wöchentliche Nachrichten und Anzeigen“ ein Intelligenz- und Anzeigeblatt nach dem Vorbild der englischen Offices of Intelligence war ${ }^{16}$ oder die Rezensionszeitung „Thornische Nachrichten von gelehrten Sachen“, die sich - laut Katarzyna Chlewicka - deutlich in Distanz zu den von Lessing herausgegeben „Briefen, die neuste Literatur betreffend" positioniert. ${ }^{17}$ Den Band ergänzen interessante Beiträge über mikrohistorisch relevante Ereignisse und Konflikte um den Deutschen Orden, ${ }^{18}$ über den sichtbaren Rückstand in der Entwicklung der Reisekultur in Polen des 18. Jahrhunderts, ${ }^{19}$ über Fremdwahrnehmung der Polen und der polnischen Kultur in der ersten Hälfte des 19. Jahrhunderts am Beispiel vom Reisetagebuch des Komponisten Franz Xaver Mozart ${ }^{20}$ und nicht zuletzt über den englischsprachigen Polendiskurs, dessen Erforschung allerdings noch weitgehend aussteht. ${ }^{21}$ Viele Beiträge dieses Bandes verweisen sichtbar auf noch bestehende Lücken in der Erforschung der deutsch-polnischen Kulturkontakte in der Frühen Neuzeit und können für weitere komparatistische Untersuchungen aufschlussreich sein.

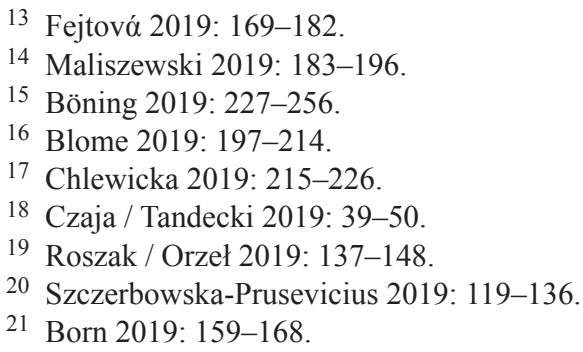


Damit gewinnt der ganze Band eine über die traditionelle und topische Zeitgebundenheit einer Gelegenheitsschrift hinausgehende Dimension.

\section{Literatur}

Adam, Wolfgang / Westphal, Siegrid (Hrsg.) in Verbindung mit Sittig, Claudius / Siebers, Winfried (2012): Handbuch kultureller Zentren der Frühen Neuzeit. Städte und Residenzen im alten deutschen Sprachraum, 3 Bde. Berlin/Boston.

Blome, Astrit (2019): Von Geborenen, Gestorbenen und Verehelichten - demographischer Wandel und soziale Beziehungen in den ,,Thornischen Wöchentlichen Nachrichten und Anzeigen ". In: Reisen, wahrnehmen, kommunizieren. a.a.O. S. 197-214.

Born, Jan (2019): Polen aus englischsprachiger Sicht am Beispiel von Michael Morans Reisebericht "A Country in the Moon: Travels in search oft he heart of Poland" (2008). In: Reisen, wahrnehmen, kommunizieren. a.a.O. S. 159-168.

Böning, Holger (2019): Konfliktlinien in den Zeitungsnachrichten im Vorfeld des Dreißigjährigen Kriegs und das Bild Polens in der Zeitungsberichterstattung des Jahres 1609. In: Reisen, wahrnehmen, kommunizieren. a.a.O. S. 227-256.

Bovt, Aleksandra (2019): Körperpflege in Polen-Litauern aus der Sicht der Reisenden. Im Spannungsverhältnis zwischen Eigenem und Fremden. In: Reisen, wahrnehmen, kommunizieren. a.a.O., S. 63-70.

Chlewicka, Katarzyna (2019): „Es ist so gefährlich ein Schriftsteller als ein Journalist zu werden. “ Über die Maßstäbe des angemessenen Rezensierens in den „Thornischen Nachrichten von gelehrten Sachen “ (1726-1766). In: Reisen, wahrnehmen, kommunizieren. a.a.O. S. 215-226.

Czubański, Antoni / Kulak, Zbigniew (1987): Ślask i Pomorze w stosunkach polsko-niemieckich od XVI do XVIII w., XIV Konferencja Wspólnej Komisji Podręcznikowej PRL-RFN Historyków. Poznań.

Czaja, Roman / Tandecki, Janusz (2019): Rechtsverkehr zwischen dem Ordensland und Magdeburg in den Auseinandersetzungen zwischen den preußischen Städten und dem Deutschen Orden in der ersten Hälfte des 15. Jahrhunderts. In: Reisen, wahrnehmen, kommunizieren. a.a.O. S. 39-50.

Czarnecka, Mirosława (2012): Breslau. In: Adam, Wolfgang / Westphal, Siegrid (Hrsg.) (in Verbindung mit Claudius Sittig und Winfried Siebers): Handbuch kultureller Zentren der Frühen Neuzeit. Städte und Residenzen im alten deutschen Sprachraum, 3 Bde, hier Bd. 1. Berlin. S. 197-238.

Dumowski, Jarosław (2019): Bouillon en poche-altpolnische Kochrezepte für Reisende. In: Reisen, wahrnehmen, kommunizieren. a.a.O. S. 39-48.

Fejtovó, Olga (2019): Das Bild Polens im Historischen Kalender von Daniel Adam Veleslavin. In: Reisen, wahrnehmen, kommunizieren. a.a.O. S. 169-182.

Lambrecht, Karen (2000): Breslau als Zentrum der gelehrten Kommunikation unter Bischof Johnn V. Thurzo (1466-1520). In: Archiv für schlesische Kirchengeschichte 58, S. 117-141.

Kizik, Edmund (2019): Die Gäste in der Festung Weichselmünde bei Danzig im 17. und 18. Jahrhundert. In: Reisen, wahrnehmen, kommunizieren. a.a.O. S. 71-78.

Kodzik, Joanna (2019): Im Wirtshaus und in der Postkutsche. Herrnhuttische Kommunikationsstrategien auf Reisen am Beispiel Polen-Litauens. In: Reisen, wahrnehmen, kommunizieren. a.a.O. S. 79-98.

Kopiński, Krzysztof (2019): Zwischen Mobilität, kaufmännischen Strategien und Migration am Beispiel des Thorner Patriziats des 14. und 15. Jahrhunderts. In: Reisen, wahrnehmen, kommunizieren. a.a.O. S. 19-28.

Maliszewski, Kazimierz (2019): Theatrum mundi der Barockzeit. Die Welt in handgeschriebenen Zeitungen Polen-Litauens. In: Reisen, wahrnehmen, kommunizieren. a.a.O. S. 183-196. 
Reisen, wahrnehmen, kommunizieren, a.a.O.

Roszak, Stanislaw / Orzeł, Joanna (2019): Lesen statt Erleben. Über Beschränkungen beim Reisen in der ersten Hälfte des 18. Jahrhunderts. In: Reisen, wahrnehmen, kommunizieren. a.a.O. S. $137-148$.

Segebrecht, Wulf(1977): Das Gelegenheitsgedicht. Ein Beitrag zur Geschichte und Poetik der deutschen Lyrik. Stuttgart.

Szczerbowska-Prusevicius, Katarzyna (2019): Franz Xaver Wolfgang Mozarts Aufenthalt in Warschau im Lichte seines Reisetagebuchs. In: Reisen, wahrnehmen, kommunizieren. a.a.O. S. 119-136.

Szyrocki, Marian (1964): Andreas Gryphius. Sein Leben und Werk, Tübingen.

Walter, Axel E. (2019): Übernachten auf Reisen, - Anmerkungen zu einem zentralen Aspekt der frühneuzeitlichen Reisekultur. In: Reisen, wahrnehmen, kommunizieren. a.a.O. S. 51-62.

Viragello, George (1988): Wasser und Seife, Puder und Parfüm. Geschichte der Körperhygiene seit dem Mittelalter. Frankfurt am Main.

Wotschke, Theodor (1939): Schlesier auf dem Thorner Gymnasium im 17. Jahrhundert. In: Zeitschrift des Vereins für Geschichte und Alterthum Schlesiens LXXIII, S. 196-216.

Zientara, Włodzimierz (1996): Die schlesischen Lehrer und Schüler an den Akademischen Gymnasien Königlich Preußens in der ersten Hälfte des 18. Jahrhunderts. In: Germanica Wratislaviensia 94, S. 215-222.

Zientara, Włodzimierz (2003): Sarmatia Europiana oder Sarmatia Asiana? Polen in den deutschsprachigen Druckwerken des 17. Jahrhunderts. Torun.

Mirostawa Czarnecka

(Universität Wrocław, Wrocław)

ORCID: 0000-0001-5226-5260

Mirosława Czarnecka, Uniwersytet Wrocławski, Instytut Filologii Germańskiej, pl. biskupa Nankiera 15b, 50-140 Wrocław, Polen, E-Mail: miroslawa.czarnecka@uwr.edu.pl.

Received: 25.08.2020, accepted: 2.03.2021

Ulrike Gleixner, Constanze Baum, Jörn Münkner, Hole Rößler (Hrsg.): Biographien des Buches (Kulturen des Sammelns. Akteure-Objekte-Medien, Bd. 1), Wallstein, Göttingen 2017, 477 S., 141 Abb.

https://doi.org/10.19195/0435-5865.146.15

Mit den „Kulturen des Sammelns“ initiiert die Herzog August Bibliothek Wolfenbüttel (HAB) eine neue Reihe mit dem Ziel, das Phänomen des Sammelns als einen zusammengesetzten Kulturprozess zu präsentieren und ihn in historischer und theoretischer Hinsicht zu untersuchen. Der erste Band der Reihe „Biographien des Buches“ lenkt die Aufmerksamkeit auf das gedruckte Medium Buch und die Handschrift, wobei „das Interesse vor allem den Spuren der Interaktion und den Transformationen, Bedeutungsveränderungen, Umwidmungen, Umarbeitungen und Vernutzungen [gilt], die an den Büchern sichtbar werden" (Biographien des Buches, S. 12).

Noch im ersten Jahrzehnt des 21. Jahrhunderts war der Begriff „Objektbiographie“ in der deutschen Wissenschaftssprache kaum verbreitet. ${ }^{1}$ Er leitet sich von der Empirischen

1 Braun 2015: 9. 